\title{
Atletas de basquetebol universitário: uma proposta de intervenção psicológica no lance livre
}

\author{
University basketball athletes: a psychological intervention proposal on the free throw
}

\author{
Bruna Feitosa de Oliveira ${ }^{1, *}$ \\ André Luis Aroni ${ }^{1,2}$ \\ Kauan Galvão Morão ${ }^{1}$ \\ Renato Henrique Verzani ${ }^{1}$ \\ Guilherme Bagni ${ }^{1,3}$ \\ Afonso Antonio Machado ${ }^{1}$
}

\begin{abstract}
Resumo
Objetivo: O objetivo do estudo foi analisar a eficiência de um programa de 8 semanas focado em exercícios respiratórios como técnica de relaxamento antes do treinamento de lance livre, visando aprimorar o desempenho neste fundamento. Métodos: Participaram 9 atletas universitárias de basquetebol feminino, com idades entre 20 e 26 anos e pelo menos 2 anos de prática. Utilizou-se como instrumento o biofeedback para visualizar alterações nos parâmetros fisiológicos periféricos das atletas, e relacioná-los com seus estados emocionais, mensurando: frequência cardíaca, frequência e volume respiratório e condutibilidade da pele. Os testes inicial e final consistiam em 3 séries de 5 lances livres. As atletas foram divididas aleatoriamente em 2 grupos, controle e intervenção, e ambos treinaram durante 8 semanas 3 séries de 5 arremessos, mas somente o grupo intervenção recebeu um treino específico de como utilizar a respiração diafragmática na rotina antes do arremesso. Resultados: Não foram encontrados padrões de acertos entre os grupos, porém nem todas as atletas do grupo intervenção utilizaram a rotina no teste final, o que pode estar relacionado com o curto espaço de tempo destinado à aquisição de novas habilidades psicológicas, inviabilizando que a rotina fosse assimilada pelas atletas. Portanto, o objetivo de aprimorar o desempenho das atletas em 8 semanas não obteve sucesso. Conclusão: Sugere-se um tempo maior de intervenção para que a rotina seja automatizada.
\end{abstract}

Palavras-chave: psicologia do esporte, biorretroalimentação psicológica, desenvolvimento humano, emoções.

\begin{abstract}
Objective: This study aims to analyze the effectiveness of an eight-weekprogram focused on breathingexercises, such as a relaxationstrategy prior to free-throw training, seekingimprovement in the performance of basketballathletes. Methods: Nine femalebasketballfemaleathleteshadparticipated, with ages between 20 and 26 years and atleasttwoyears of competitivepractice. Biofeedback was used as an instrument to visualize changes in the peripheralphysiologicalparameters of the athletes, and to relate them to their emotionalstates, measuring: heart rate, respiratory rate and volume, and skinconductance. The initial and final testconsisted of three sets of fivefreethrows. The athleteswererandomlydividedintotwogroups, control and intervention, both of whomtrained the three sets of fivepitches for eightweeks, but the intervention groupalsoreceivedspecific training, trying to use diaphragmaticbreathing in the routine that precedes the throw. Results: No match patternswerefoundbetween the groups, but not all the athletes in the intervention group used the routine in the final test, which may be related to the short time for an acquisition of new psychologicalskills, making it difficult for athletes to assimilate their routines. Therefore, the aim of the study in developingathletes' performance in eightweeks was not successful. Conclusion: Alonger intervention time is suggested to automatize the routine.
\end{abstract}

Key-words: sports psychology, biofeedback psychology, human development, emotions.
Afiliação dos autores

${ }^{1}$ Laboratório de Estudos e Pesquisa em Psicologia do Esporte

(LEPESPE), Universidade Estadual Paulista, Rio Claro, São Paulo, Brasil.

${ }^{2}$ DeVry/Metrocamp, Campinas, São

Paulo, Brasil.

${ }^{3}$ Fundação Hermínio Ometto,

Uniararas, Araras, São Paulo, Brasil.

*Autor correspondente

Av. 24A, 1515, Bela Vista, Rio Claro, SP, Brasil.

e-mail:

brunaffeitosa@hotmail.com

Conflito de interesses

Os autores declararam não haver conflito de interesses.

Processo de arbitragem

Recebido: 22/11/2017 Aprovado: 20/01/2018 


\section{Introdução}

O contexto esportivo consiste em um ambiente propício para o surgimento de emoções, com diversas motivações e intensidades ${ }^{1}$. Estas emoções podem favorecer ou não o desempenho de um atleta durante o momento da competição, e por esta razão é muito importante identificá-las e administrá-las. $\mathrm{E}$ dentre todas as emoções que podem ser vivenciadas pelos atletas durante o período competitivo, as mais comumente citadas são ansiedade, medo e vergonha ${ }^{2,3}$.

No basquete existem muitas situações que despertam diversas emoções, dentre elas o lance livre, que é considerado uma ótima oportunidade para conquistar pontos, podendo, muitas vezes, decidir quem vencerá a partida ${ }^{4,5}$. Atletas do alto nível competitivo apresentam habilidades técnicas bastante apuradas, mas, ainda assim, o número de erros em lances livres aumenta em momentos críticos do jogo, como, por exemplo, fim da partida, importância do torneio ou resultado esportivo. Por esta razão, torna-se fundamental considerar o estado emocional dos atletas como possível razão para a queda do desempenho.

Com intuito de administrar estes estados emocionais de forma mais eficiente, já foram propostas algumas técnicas que podem ser aplicadas com os atletas durante o treinamento da modalidade, tais como definição de metas, relaxamento, mentalização e auto conversa ${ }^{6}$. As técnicas de relaxamento promovem uma redução dos níveis de ansiedade dos atletas ${ }^{7}, 0$ que pode contribuir para que eles potencializem a execução das tarefas as quais já estão habituados. A relação entre os níveis de ansiedade e o desempenho apresentado foi descrita por Vasconcelos-Raposo ${ }^{8}$, que afirma que o desempenho do atleta pode ser influenciado tanto positivamente quanto negativamente pela mesma, dependendo da intensidade experimentada pelo indivíduo. Deste modo, reduzir os níveis de ansiedade pode ser importante em momentos de grande tensão da partida, e esta é uma das finalidades em que as técnicas de relaxamento têm sido utilizadas no contexto esportivo.

Como exemplo dessas técnicas, Weinberg e Gould ${ }^{7}$ citam: relaxamento progressivo, que consiste no processo de contrair e relaxar grupos musculares específicos progressivamente; controle da respiração, que se baseia em respirar de forma profunda utilizando o músculo diafragma; biofeedback, que é uma ferramenta que detecta parâmetros fisiológicos enquanto o atleta realiza uma ação; resposta de treinamento, que consiste em exercícios de meditação; treinamento autógeno, entre outros.

Outra técnica frequentemente sugerida é a mentalização, que pode ser definida como o processo de criar ou recriar experiências mentalmente, sem realizar estas ações de fato. Estudos recentes demonstraram que esta técnica contribui para um aumento da confiança, concentração e enfrentamento da ansiedade, o que reforça sua utilização em situações de grande tensão durante uma partida ${ }^{9,10}$

Outra importante estratégia utilizada para aumentar a concentração dos atletas é a rotina de desempenho, que pode ser definida como uma técnica individualizada que une uma série de pensamentos e ações padrão a serem realizadas pelo atleta antes da tarefa propriamente dita ${ }^{11}$. Esta técnica direciona o foco de atenção do atleta para a tarefa que deve ser realizada, ao mesmo tempo em que o desvia de pensamentos negativos e/ou estímulos externos. Esta estratégia pode, ainda, reforçar a confiança do atleta e diminuir sua ansiedade, visto que a rotina de desempenho limita a variabilidade dos movimentos, o que consequentemente aumenta as chances de realizar a ação de forma eficiente ${ }^{12,13}$.

Outro fator que pode interferir na efetividade desta técnica é a mudança de padrões da rotina. Se um atleta está habituado a realizar um grupo de ações antes do lance livre e em um dado momento é solicitado que ele altere este padrão pode haver uma queda de desempenho até que o mesmo se acostume com o novo padrão ${ }^{13}$.

Todas as técnicas mencionadas acima têm como um dos objetivos principais aumentar a concentração, a qual pode ser caracterizada por quatro comportamentos principais: focalizar os aspectos mais relevantes do ambiente, manter o foco de atenção durante um tempo, ter consciência da situação e mudar o foco de atenção quando necessário ${ }^{7}$. Esta habilidade mental é frequentemente citada como uma prática constante de atletas talentosos, independente do esporte ou tarefa realizada. Segundo Phelps e Kulinna ${ }^{13}$ esta capacidade é especialmente relevante na execução de tarefas de cadeia fechada, tal como o lance livre, uma vez que seus resultados podem ser fortemente afetados por distrações internas e externas. Por esta razão é de grande interesse que os atletas encontrem meios de desenvolver esta capacidade a fim de alcançar resultados mais expressivos.

Especificamente no lance livre, a aplicação de rotinas psicológicas e de desempenho, tais como relaxamento, auto conversa positiva e reprodução de movimentos repetitivos, parece estar relacionado com um número maior de acertos ${ }^{13,12}$.

Entretanto, parece haver diferenças com relação ao tempo que essas estratégias devem ser aplicadas para surtir um efeito positivo. Estudos como o de Lamirand e Rainey ${ }^{14}$ e Kanthack et al. ${ }^{15}$, evidenciaram que, a curto prazo, as técnicas de relaxamento apresentaram resultados mais significativos, se comparadas às técnicas de mentalização. Isso se deve ao fato de que a mentalização costuma apresentar resultados mais expressivos quando aplicadas em longo prazo, como sugere o estudo de Schuster et al. ${ }^{16}$. Mas vale ressaltar que, quando aliadas as duas técnicas, os benefícios encontrados são ainda mais expressivos, se comparados à aplicação das técnicas de forma separada. De qualquer forma, ainda há dúvida sobre o período de tempo ideal para intervenções desse tipo.

Entretanto existem alguns aspectos que podem influenciar a efetividade desta técnica. Foi destacado por Czech, Ploszay e Burke $^{12}$ em seu estudo que o contexto pode ter afetado a rotina dos atletas observados, isto é, situações como placar desfavorável ou proximidade do fim da partida podem ter tido influência na realização da rotina e, consequentemente, no desempenho dos lances livres. Ainda, os autores destacaram que em alguns momentos os atletas estavam tão preocupados com o resultado final da ação que não dedicavam atenção necessária para realizar a rotina.

Weinberg e Gould ${ }^{7}$ indicam que para um atleta, em seu primeiro contato, são necessários entre 3 e 6 meses de treinamento, para aquisição de novas habilidades psicológicas. Ressalta-se que diferentes variáveis podem influenciar nesse processo, principalmente o nível do atleta. Os autores destacam que tais habilidades devem, portanto, serem partes integrantes da periodização da modalidade.

Com base nas informações acima, o objetivo desta pesquisa foi analisar a eficiência de um programa de oito semanas focado em exercícios respiratórios, como estratégia de relaxamento antes do treinamento de lance livre, buscando melhoria no desempenho de atletas de basquetebol.

\section{Métodos}

Esta foi uma pesquisa mista de caráter avaliativo. $\mathrm{O}$ foco desse tipo de pesquisa é a utilização de dados para avaliar uma determinada prática ou programa, movimento ou evento ${ }^{17}$. O estudo também apresentou uma característica experimental, que é definido por Rampazzo ${ }^{18}$ como um tipo de pesquisa na qual se manipula variáveis específicas a fim de criar a situação que se quer estudar

As participantes do estudo foram nove atletas do time de basquetebol feminino da Universidade Estadual Paulista, localizada na cidade de Rio Claro/SP. Todas possuíam mais de dois anos de experiência competitiva na modalidade $(M=9 / D P$ $=4,79$ ), e idades compreendidas entre os vinte e vinte e seis anos $(M=23,6 / D P=3,3)$. Todas as normas éticas indicadas pela Declaração de Helsinki (1975, revisada em 1983), além das Resoluções do Conselho Nacional de Saúde n. 196, de 10/10/96 e n. 251 , de $07 / 08 / 1997$, foram seguidas nesse estudo. O projeto de pesquisa foi analisado e aprovado pelo Comitê de Ética em Pesquisa (CEP) em Seres Humanos do Instituto de Biociências da UNESP-Rio Claro/SP, parecer número 1.815.375.

O biofeedback, mais especificamente o Nexus-10, foi utilizado para visualizar alterações nos parâmetros fisiológicos periféricos das atletas, e relacioná-los com seus estados emocionais $^{19}$. O estudo se concentrou em quatro parâmetros: frequência cardíaca $(F C)$, frequência $(F R)$ e volume respiratório (VR) e condutibilidade da pele (CP). Com relação à FC e FR, espera-se que valores mais baixos facilitem o gesto motor. Já a CP pode evidenciar o "estresse" atual da atleta, valores mais altos apontam menor probabilidade de boa performance.

Em um dia previamente agendado com as nove atletas, foi realizado o teste de desempenho que ocorreu antes de uma sessão de treino. Antes de iniciar, todas assinaram o Termo de 
Consentimento Livre e Esclarecido. Como primeira tarefa, elas deveriam realizar três séries de cinco lances livres, com pausas de trinta segundos entre os arremessos, sendo a quantidade de acertos registrada em um caderno de anotações. Antes do primeiro arremesso, coletamos com o Nexus-10 os dados relacionados ao estado fisiológico periférico das atletas durante um minuto em repouso. Neste momento, dividimos aleatoriamente as atletas em dois grupos distintos: um de controle, que treinou as três séries de arremessos durante as oito semanas seguintes; o segundo grupo recebeu treinamento específico de como utilizar a respiração diafragmática na rotina que antecede 0 arremesso, repetindo também durante oito semanas as três séries de cinco arremessos. Ao fim da oitava semana repetimos o teste inicial, com o objetivo de avaliar o desempenho esportivo e fisiológico das atletas. A seguir, comparamos a evolução das atletas entre o primeiro e último teste de forma individual.

\section{Resultados e Discussão}

Com base no que foi proposto nos procedimentos metodológicos, foi realizada uma análise individual das atletas em dois momentos distintos, levando-se em consideração a existência de um grupo intervenção e de um grupo controle, isto é, no momento do teste inicial todas as atletas tiveram coletadas a FC basal, a FC antes de cada uma das três séries, bem como o número de acertos nessas.

Com isso, o grupo intervenção (formado por quatro atletas) passou a realizar e treinar uma rotina (criada por cada uma delas) antes de cada arremesso livre. Nessa rotina, foi pedido que realizassem uma única respiração profunda (diafragmática) com o objetivo de buscar maior relaxamento para a ação. O processo de treino durou oito semanas, neste tempo, o grupo controle seguiu os treinos de lance livre normalmente sem se preocupar com rotinas e respiração. Ambos os grupos realizaram a mesma tarefa após oito semanas, mantendo a mesma ordem de atletas do teste inicial. Desta maneira, os resultados estão expostos a seguir, em tabelas que facilitam a visualização dos dados encontrados individualmente em ambos os momentos:

Tabela 1

Frequências cardíacas basais e por séries.

\begin{tabular}{|c|c|c|c|c|c|c|c|c|}
\hline \multirow{2}{*}{ Atleta } & \multicolumn{2}{|c|}{ FC Basal (bpm) } & \multicolumn{2}{|c|}{ FC antes da $1^{\text {a }}$ série $(\mathrm{bpm})$} & \multicolumn{2}{|c|}{ FC antes da $2^{\mathrm{a}}$ série (bpm) } & \multicolumn{2}{|c|}{ FC antes da $3^{a}$ série $(\mathrm{bpm})$} \\
\hline & Pré & Pós & Pré & Pós & Pré & Pós & Pré & Pós \\
\hline Atleta 1 (I) & 85 & 74 & 104 & 92 & 93 & 94 & 103 & 88 \\
\hline Atleta 2 (I) & 108 & 85 & 143 & 107 & 125 & 83 & 124 & 94 \\
\hline Atleta 3 (C) & 99 & 80 & 113 & 90 & 101 & 83 & 107 & 95 \\
\hline Atleta 4 (I) & 110 & 66 & 134 & 84 & 125 & 90 & 116 & 89 \\
\hline Atleta $5(\mathrm{C})$ & 91 & 86 & 107 & 103 & 101 & 87 & 98 & 86 \\
\hline Atleta 6 (C) & 108 & 85 & 136 & 119 & 136 & 110 & 129 & 124 \\
\hline Atleta 7 (I) & 82 & 65 & 107 & 86 & 95 & 87 & 91 & 79 \\
\hline Atleta 8 (C) & 79 & 97 & 113 & 122 & 93 & 131 & 99 & 109 \\
\hline Atleta $9(\mathrm{C})$ & 91 & 105 & 123 & 122 & 111 & 132 & 118 & 139 \\
\hline Grupo Intervenção* & 96,25 & 72,5 & 122 & 92,25 & 109,5 & 88,5 & 108,5 & 87,5 \\
\hline Grupo Controle* & 93,6 & 90,6 & 118,4 & 111,2 & 108,4 & 108,6 & 110,2 & 110,6 \\
\hline
\end{tabular}

* Média dos grupos. Obs.: (I) corresponde ao grupo intervenção e (C) ao grupo controle.

Na tabela 1 observamos os resultados por atletas e, ao final, as médias de ambos os grupos quanto a cada um dos momentos em que foi coletada a FC das participantes. Como esperado, os resultados encontrados na FC basal foram menores em todas as atletas quando comparados aos momentos em que executavam a primeira série da rotina, reforçando a confiabilidade do instrumento utilizado nesta pesquisa para analisar este fator. Essa comparação justifica-se pelo fato da participante partir do repouso absoluto para o exercício, pois da segunda série em diante, as atletas já poderiam ter adotado estratégias como as ensinadas na rotina para o controle da FC. De acordo com Berridge e Winkielman ${ }^{20}$, as pessoas podem não ser tão precisas ao comunicar suas percepções afetivas em algumas circunstâncias, entretanto, algumas reações fisiológicas podem revelar alterações emocionais, como a FC.

Com exceção de duas atletas pertencentes ao grupo controle, todas as outras apresentaram valores mais elevados na FC basal no teste inicial em relação a basal teste final, o que pode ter relação com o fato de que no teste final todas já haviam vivenciado o procedimento que seria adotado ao longo da coleta, gerado assim uma alteração comportamental. Acevedo e
Ekkekakis ${ }^{21}$ confirmam a evidência que alterações psicológicas são comumente seguidas de alterações fisiológicas, a FC neste caso. De qualquer forma, tanto o fato de algumas atletas terem demonstrado alterações na FC na comparação entre teste inicial e final, quanto as que não evidenciaram alterações, pode ser explicado pela afirmação de $\operatorname{Hanin}^{22,23}$, que ressalta que estados fisiológicos são idiossincráticos, ou seja, particulares em cada pessoa ou atleta. Isso torna ainda mais relevante a tentativa de identificar as zonas de ótimo rendimento em cada atleta em processos de intervenção.

Ao analisar os grupos, é possível perceber que no grupo intervenção, todas as FC do teste final obtiveram resultados menos elevados do que no teste inicial, o que não é uma realidade observada no grupo controle. No grupo intervenção, no teste final, também ficou claro que os valores das médias foram reduzindo de série para série, isto é, com o maior valor na primeira série e o menor valor na última série, algo que, entre inúmeras variáveis, também pode estar atrelado à intervenção proposta.

A tabela a seguir (Tabela 2) conta com os números envolvendo os acertos das atletas nos cinco arremessos realizados e dos grupos (intervenção e controle).

Tabela 2

Número de acerto das atletas.

\begin{tabular}{|c|c|c|c|c|c|c|c|c|}
\hline \multirow{2}{*}{ Atleta } & \multicolumn{2}{|c|}{ Acertos $1^{\text {a }}$ série } & \multicolumn{2}{|c|}{ Acertos $2^{a}$ série } & \multicolumn{2}{|c|}{ Acertos 3as série } & \multicolumn{2}{|c|}{ Médias } \\
\hline & Pré & Pós & Pré & Pós & Pré & Pós & Pré & Pós \\
\hline Atleta 1 (I) & 2 & 2 & 3 & 2 & 1 & 4 & 2 & 2,67 \\
\hline Atleta 2 (I) & 4 & 2 & 2 & 3 & 2 & 3 & 2,67 & 2,67 \\
\hline Atleta 3 (C) & 3 & 2 & 5 & 2 & 4 & 1 & 4 & 1,67 \\
\hline Atleta 4 (I) & 2 & 1 & 2 & 0 & 3 & 2 & 2,33 & 1 \\
\hline Atleta 5 (C) & 2 & 2 & 5 & 3 & 5 & 2 & 4 & 2,33 \\
\hline Atleta 6 (C) & 4 & 4 & 2 & 4 & 2 & 4 & 2,67 & 4 \\
\hline Atleta 7 (I) & 1 & 0 & 3 & 2 & 3 & 3 & 2,33 & 1,67 \\
\hline Atleta 8 (C) & 1 & 4 & 2 & 3 & 1 & 3 & 1,33 & 3,33 \\
\hline Atleta 9 (C) & 1 & 0 & 0 & 4 & 2 & 3 & 1 & 2,33 \\
\hline Grupo (I)* & 2,25 & 1,25 & 2,5 & 1,75 & 2,5 & 3 & 2,33 & 2,33 \\
\hline Grupo $(C)^{*}$ & 2,2 & 2,4 & 2,8 & 3,2 & 2,8 & 2,6 & 2,6 & 2,73 \\
\hline
\end{tabular}

Ao contrário do que ocorreu em relação à $\mathrm{FC}$, ao analisar os valores de acertos dos grupos, não foram encontrados padrões de respostas entre eles. Observando o grupo intervenção, temos que duas das atletas diminuíram a média de acertos no teste final, enquanto uma manteve a média e a outra melhorou o rendimento. No grupo controle, por sua vez, três das participantes aumentaram a média de acertos e duas diminuíram. 
Contudo, ao analisar as atletas do grupo de intervenção, procurou-se verificar se as atletas estavam utilizando a respiração diafragmática durante o teste final, de acordo com o que foi pedido durante a intervenção, e assim, percebeu-se ao analisar o comportamento respiratório pelo biofeedback que duas mantiveram uma rotina adequada (Atletas 1 e 2 ), enquanto a atleta 4 mau utilizou a respiração diafragmática e a atleta 7 oscilou entre as séries (ver Figura 1). Neste ponto é importante destacar que os valores de frequência e volume respiratório não foram avaliados, visto que o objetivo era analisar a utilização de um comportamento adequado de respiração diafragmática, entre a inspiração e expiração. Este fato pode estar relacionado às alterações nas médias de acertos e pode ser justificado, talvez, pela preocupação de algumas atletas em tentar melhorar seu desempenho prévio, o que pode ter acarretado na redução do foco na rotina que deveria ter sido realizada. Lidor ${ }^{24}$ relata que atletas profissionaispossuem rotinas bem definidas, já no caso de atletas amadores (atletas deste estudo), a rotina pode ainda não ser automática em inúmeras situações de jogo, e até dificultar o gesto técnico.

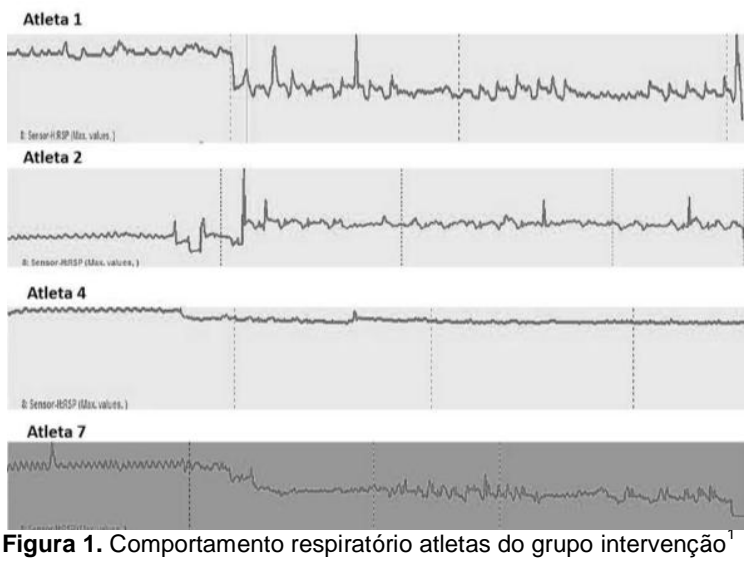

Já com relação à média dos grupos, nota-se que os resultados do grupo intervenção foram menores no teste final nos acertos das primeiras e segundas séries das participantes, sendo maior apenas na terceira série de arremessos. Já no grupo controle ocorreu o inverso, com melhores desempenhos nas duas primeiras séries no teste final e menor na terceira série. Contudo, nas médias envolvendo as três séries, o grupo intervenção manteve o valor de 2,33 e o grupo controle melhorou, partindo de 2,6 para 2,73. De volta a afirmação de Lidor $^{24}$, no caso das atletas deste estudo, a intervenção de oito semanas não foi o suficiente para gerar melhora na performance esportiva pós teste, o que pode ser justificado pelo fato de atletas amadores não estarem acostumadas a realizar rotinas de forma eficiente. Se observarmos o rendimento individual no grupo intervenção (Tabela 2), podemos perceber que nos casos das atletas 4 e 7 , houve uma queda no desempenho pós teste. Este dado reforça a literatura ${ }^{24}$ no sentido da importância de se utilizar rotinas e as habilidades psicológicas básicas em diversos esportes ${ }^{6}$, porém, o timing para a consolidação e aumento de rendimento pode depender de cada atleta ${ }^{22}$. Neste sentido, voltamos a reforçar a afirmação de Weinberg e Gould ${ }^{7}$, indicando a necessidade de 3 a 6 meses de treinamento para a aquisição de novas habilidades psicológicas. Portanto, o objetivo do estudo em desenvolver o rendimento das atletas em oito semanas, não teve sucesso.

A tabela 3 conta com os resultados envolvendo a condutibilidade das atletas nos períodos pré e pós-intervenção, bem como as médias dos grupos intervenção e controle.

Observando as atletas do grupo intervenção, em relação à condutibilidade nestes dois momentos, temos que três das quatro atletas diminuíram os valores desta variável fisiológica, enquanto no grupo controle três apresentaram diminuição da condutibilidade. Por outro lado, observando as médias de ambos os grupos, constatou-se que o grupo intervenção revelou no teste final a manutenção da média encontrada no teste inicial, enquanto o grupo controle obteve diminuição deste valor.

${ }^{1}$ Nota: as imagens retratam o comportamento respiratório das atletas durante o tempo de execução das séries. Assim, cada pico registrado no gráfico representa a inspiração, enquanto a base mostra o momento de expiração máxima.
Mesmo com esse dado, seria arriscado presumir que essas informações justificariam tais desempenhos entre os grupos, pois há inúmeras variáveis intervindo no contexto estudado.

Tabela 3

Condutibilidade das atletas

\begin{tabular}{ccc}
\hline Atleta & $\begin{array}{c}\text { Condutibilidade } \\
\text { Pré-teste }\end{array}$ & $\begin{array}{c}\text { Condutibilidade } \\
\text { Pós-teste }\end{array}$ \\
\hline Atleta 1 (I) & 1,16 & 1,06 \\
Atleta 2 (I) & 1,31 & 1,45 \\
Atleta 3 (C) & 10,43 & 7,76 \\
Atleta 4 (I) & 6,64 & 5,5 \\
Atleta 5 (C) & 2,28 & 2,47 \\
Atleta 6 (C) & 10,83 & 4,4 \\
Atleta 7 (I) & 3,88 & 1,94 \\
Atleta 8 (C) & 1,54 & 0,7 \\
Atleta 9 (C) & 2,81 & 6,28 \\
\hline Média dos grupos. Obs.: (I) corresponde ao grupo intervenção e (C) ao grupo \\
controle.
\end{tabular}

De qualquer forma, é importante frisar que nos últimos quinze anos muitos estudos focaram seus esforços em definir os picos de performance de atletas, assim como identificar as zonas de rendimentos ótimo e não ótimos durante Torneios e Competições, a fim de definir estratégias de treino, jogo ou prova. Um bom exemplo é o trabalho e Kamata, Tenenbaum e Hanin $^{25}$ onde os autores relatam a probabilidade do bom rendimento esportivo entre zonas de baixa ativação até zonas de elevado estresse e excitação fisiológica. No caso das atletas desse estudo, seria importante trabalhar de forma longitudinal a fim de compreender suas melhores zonas de rendimento no lance livre, para depois analisarmos se as alterações de condutibilidade que tiveram as conduziram ao bom ou mau desempenho.

A tentativa de verificar se em um curto espaço de tempo as atletas já apresentariam melhoras no desempenho não foi alcançada, possivelmente devido ao fato das rotinas não terem, ainda, sido assimiladas, indo de encontro ao que Lidor $^{24}$ e Weinberg e Gould ${ }^{7}$ apontam sobre o período necessário para a aquisição de novas habilidades psicológicas.

\section{Conclusão}

Este estudo teve como objetivo analisar o desempenho de atletas universitárias de basquetebol durante o lance livre. De forma mais específica, buscou-se analisar a eficiência de um programa de oito semanas focado em exercícios respiratórios, como uma estratégia de relaxamento antes dos arremessos e, consequentemente, aumento de rendimento esportivo. Com relação à performance, não se observou evolução após a intervenção, contudo, algumas variáveis foram destacadas sobre o método de intervenção.

Destacamos que existiram algumas limitações no estudo, sendo que a principal foi o período de tempo em que se foram trabalhadas as técnicas de respiração diafragmática. Sendo assim, sugerimos novos estudos com um tempo maior para a introdução de tal técnica, bem como avaliações mais periódicas ao longo de uma temporada, verificando inclusive, o desempenho esportivo em partidas oficiais, já que o estudo não verificou o desempenho em tais eventos.

O estudo aponta para uma mudança na prática pedagógica dos futuros professores de EF, visto que os alunos usaram tanto o método tradicional (centrado na técnica), como os jogos, superando a predominância do modelo tecnicista. A utilização de apenas um método provavelmente não promoverá o sucesso no processo de ensino e aprendizagem nessa fase do desenvolvimento. Todas as abordagens possuem sua importância e, portanto, cabe aos professores conhecerem diferentes métodos, a fim de escolher os mais adequados a partir da análise das possibilidades e necessidades dos alunos, além das condições estruturais para as aulas.

Possivelmente a conclusão deste estudo tenha sido resultado de mudanças graduais no curso de graduação e do conhecimento acumulado nestes últimos anos sobre como o esporte é desenvolvido nas aulas de EF. Entretanto, outras iniciativas devem ser tomadas, com o propósito de garantir a formação didático-metodológica dos discentes, em que não ocorra apenas a transmissão destas informações, mas também a reflexão destas diversas abordagens e como resultado, se consolide uma prática docente mais alinhada às concepções pedagógicas contemporâneas. 


\section{Referências}

1. De Rose Junior D. A competição como fonte de estresse no esporte. Rev bras cien e mov 2002;10(4):19-26.

2. Dobránszky IA, Machado AA. Auto-eficácia: um estudo da sua contribuição para a avaliação de desempenho de atletas. Rev Psico USF 2001; 6:67-74.

3. Brochado MMV. O medo no esporte. Motriz 2002;8:69-77.

4. Cedra C, Sério TMAP. O treinamento do lance livre no basquetebol. Rev bras psicol esporte 2008;2(1)

5. Almas SP. Análise das estatísticas relacionadas ao jogo que discriminam as equipes vencedoras das perdedoras no basquetebol profissional brasileiro. Rev bras educ fís esporte 2015; 29:551-558.

6. Greenspan MJ, Feltz DL. Psychological Interventions With Athletes in Competitive Situations: A Review.The sport psychol 1989;3(3):219-236.

7. Weinberg RS, Gould D. Fundamentos da Psicologia do Esporte e do Exercício. 6. ed. Porto Alegre: Ed. Artmed; 2017.

8. Vasconcelos-Raposo J. Explorando as limitações do conceito de ansiedade no desporto. Rev Desporto da UTAD 2000; 1:47-66.

9. Jones $L$, Stuth $G$. The uses of mental imagery in athletics: An overview. Appl \& Prev Psychol 1997;6:101-115.

10. Godtsfriedt J, Andrade A, Vasconcelos DIC. Treinamento mental no tênis: revisão sistemática da literature. Rev bras ciênc esporte 2014;36(2):577-586.

11. Lee $D$. The effect of pre-shot routine on performance of a drive in golf. [Dissertação de mestrado]. Muncie: Ball State University; 2009.

12. Czech DR, Ploszay AJ, Burke KL. An Examination of the Maintenance of Preshot Routines in Basketball Free Throw Shooting. J Sport Behav. 2004; 27:323-329.
13. Phelps A, Kulinna P. Pre-performance routines followed by free throw shooting accuracy in secondary basketball players. Biomedical Human Kinetics. 2015; 7: 171-176.

14. Lamirand M, Rainey D. Mental imagery, relaxation, and accuracy of basketball foul shooting. Percept Mot Skills. 1994; 78: 1229-1230.

15. Kanthack TFD, Bigliassi M, Vieira LF, Altimari LR. Acute effect of motor imagery on basketball players' free throw performance and self-efficacy. Rev Bras Cineantropom Desempenho Hum. 2014;16: 47-57.

16. Schuster C, Hilfiker R, Amft O, Scheidhauer A, Andrews B, Butler J, et al Best practice for motor imagery: a systematic literature review on motor imagery training elements in five different disciplines. BMC Med. 2011; $9(75)$.

17. Thomas JR, Nelson JK. Métodos de pesquisa em atividade física. 3. ed. Porto Alegre: Artmed; 2002.

18. Rampazzo L. Metodologia científica: para alunos de graduação e pósgraduação. São Paulo: Loyola; 2013.

19. Danucalov MAD. A psicofisiologia e o biofeedback aplicado à Educação Física. Rev Mackenzie educ fís esporte. 2010;9:28-31.

20. Berridge KC, Winkielman P. What is an unconscious emotion? Cogn Emot. 2003:17:181-211.

21. Acevedo E, Ekkekakis P. Psychobiology of physical activity. Champaign: Human Kinetics; 2006.

22. Hanin YL. Emotions and athletic performance: individual zones of optimal functioning model. Euro Yearbook Sport Psychol. 1997;1:29-72.

23. Hanin YL. Emotions in sport. Champaign: Human Kinetics; 2000.

24. Lidor R. Preparatory Routines in Self-Paced Events. In: Tenenbaum G, Eklund RC, editores. Handbook of Sport Psychology. New Jersey: John Wiley \& Sons; 2007. p. 445 - 465

25. Kamata A, Tenenbaum G, Hanin YL. Individual zone of optimal functioning (IZOF): a probabilistic estimation. J Sport Exerc Psychol. 2002;24:189-208 